\title{
A Febrile Skin Rash May Hide Another
}

\author{
Manel Hsairi ${ }^{1,2 *}$, Fatma Hammemi ${ }^{2,3}$, Faiza Safi ${ }^{1,2}$, Amal Eleuch ${ }^{1,2}$, Makram Koubaa ${ }^{2,3}$, \\ Lamia Gargouri $^{1,2}$, Abdelmajid Mahfoudh ${ }^{1,2}$
}

\author{
${ }^{1}$ Department of Pediatrics, Pediatric \\ Emergency and Intensive Care. Hedi \\ Chaker University Hospital, Sfax, \\ TUNISIA \\ 2 Faculty of Medicine, University of \\ Sfax, TUNISIA \\ ${ }^{3}$ Infectious Diseases Department, Hedi \\ Chaker University Hospital, Sfax, \\ TUNISIA \\ * Corresponding author: \\ manelhsairi6@gmail.com
}

\begin{abstract}
Background: Measles mimics Kawasaki disease (KD) as there are many common features. It may be challenging to distinguish KD from measles. However, these two diseases may be associated. Here, we report a case of KD trigged by measles.

Case presentation: A 4-year-old girl was hospitalized for a 7-day-history of persistent fever and cough. She had a skin rash, conjunctivitis, cheilitis and cervical lymphadenopathy. Echocardiography showed suggestive signs of KD. The patient was treated with intravenous immunoglobulin relayed by aspirin. Measles serology revealed positive IgM and IgG antibodies.
\end{abstract}

Conclusion: We reported a febrile skin rash which hided another.

Keywords: children, Kawasaki disease, measles, skin rash

\section{(3) veriths}

\section{EUROPEAN JOURNAL OF BASIC MEDICAL SCIENCE}

Eur J Basic Med Sci 2019;9(1):1-4

Received: 16 Nov 2018

Accepted: 18 Jan 2019 


\section{INTRODUCTION}

Kawasaki disease (KD), also known as the mucocutaneous lymph node syndrome, is an acute febrile systemic vasculitis that commonly affects infants and young children, mostly below 5 years of age [1]. Its etiology remains unknown, but, infectious etiology was strongly suggested as initiating an abnormal immune response causing therefore, KD [2]. The diagnosis is based on clinical features and no specific diagnostic test exists nowadays. It may be very challenging to distinguish KD from other febrile infectious diseases including measles, which shares many features with KD, such as fever, skin rash, nonexudative conjunctivitis, generalised lymphadenopathy and malaise [3]. Measles, a highly contagious viral disease, should be bearded in mind in front of a child with fever and maculopapular rash, even in low incidence measles countries. Clinical differential diagnosis between KD and measles may be difficult. However, these 2 diseases may be associated, which can lead to misdiagnosis. Here, we report a rare case of KD trigged by measles.

\section{CASE PRESENTATION}

A 4-year-old asthmatic girl was hospitalized for a 7-dayhistory of persistent fever and cough. She received antibiotics (Amoxicillin) by self-medication, however, no improvement was noted. The evolution was marked by the appearance of a generalized skin rash which motivated the consultation. Physical examination on admission revealed a body temperature of $40^{\circ} \mathrm{C}$ and a generalized maculopapular rash which started 2 days ago. She had conjunctivitis, cheilitis and cervical lymphadenopathy. Her heart rate was 90 beats per minute and the auscultation of the heart revealed no significant abnormality. Her respiratory rate was 30 breaths per minute and pulmonary auscultation revealed bilateral late inspiratory crackles.

Abnormal laboratory investigations showed elevated inflammatory markers such as an elevated C-reactive protein $(23 \mathrm{mg} / \mathrm{l})$ and an elevated erythrocyte sedimentation rate $(30 \mathrm{~mm} / \mathrm{h})$. White blood cells account was normal $\left(6000 / \mathrm{mm}^{3}\right)$, however, a neutropenia was noted $\left(1260 / \mathrm{mm}^{3}\right)$. Aspartate aminotransferase concentration was two times above the upper limit of the normal level $(92 \mathrm{IU} / \mathrm{L})$, while alanine aminotransferase and renal function were normal. Chest X-ray revealed diffuse reticulonodular infiltrates.
The diagnosis of measles and KD were both suspected. Firstly, because of the current state of measles epidemic, it must be suspected in front of each fever with maculopapular rash. Although our patient received 2 doses of measles and rubella vaccine, that doesn't exclude the diagnosis of measles. Secondly, KD was suspected because of the association of a fever that has been evolving for more than 5 days and 4 additional criteria represented by cheilitis, exanthema, nonpurulent bilateral conjunctivitis and cervical lymphadenopathy.

A transthoracic echocardiography revealed hyperechogenic right coronary walls with a dilatation at $2.7 \mathrm{~mm}$. The patient was treated with intravenous immunoglobulin (IVIG) at a dose of $2 \mathrm{gr} / \mathrm{kg}$. Measles serology was performed and revealed positive IgM and IgG antibodies. The disease evolution was favourable with the occurrence of apyrexia 48 hours after initiating IVIG. The patient was discharged from the hospital and continued a low-dose of aspirin at $3 \mathrm{mg} / \mathrm{kg} /$ day. Her laboratory investigations, 15 days apart, showed an erythrocyte sedimentation rate at $18 \mathrm{~mm} / \mathrm{h}$ and a normal C-reactive protein $(8 \mathrm{mg} / \mathrm{L})$. Neutrophil and aspartate aminotransferase rates were $6230 / \mathrm{mm}^{3}$ and $28 \mathrm{IU} / \mathrm{L}$, respectively. Control echocardiography, after 1-month evolution, showed an improvement with a lumen diameter of the right coronary at 2 to $2.5 \mathrm{~mm}$ associated to barely-visible coronaries. The same dose of aspirin will be continued until the next echocardiography control, 6 months later.

\section{DISCUSSION}

We presented a case of $\mathrm{KD}$, most likely trigged by measles virus. Actually, measles cases continue to increase compared to the previous years. The World Health Organization reported that measles cases rose by $300 \%$ in the first 3 months of 2019 , compared to the same period in 2018 [4]. Due to the current epidemic measles state, the diagnosis of measles was suspected at first based on the presence of fever and skin rash. However, clinicians should be aware of the differential diagnosis including $\mathrm{KD}$, in order to avoid potential complication of delayed diagnosis and treatment. In fact, previous studies reported that the only significant difference found was mucositis and changes of the extremities criteria, significantly associated with KD [5]. However, in severely affected cases of measles, desquamation can occur, but, it is not seen in the hands and feet. Besides, Koplik spots, pathognomonic of measles, are not seen in $\mathrm{KD}$, but they are not always present in measles either [5]. 
Hsairi et al.

Table 1. Diagnostic criteria for classic Kawasaki disease [6]

The presence of fever for at least 5 days with at least 4 of the 5 following principal clinical features:

1. Erythema and cracking of lips, strawberry tongue, and/or erythema of oral and pharyngeal mucosa

2. Bilateral bulbar conjunctival injection without exudate

3. Rash: maculopapular, diffuse erythroderma, or erythema multiforme-like

4. Erythema and edema of the hands and feet in acute phase and/or periungual desquamation in subacute phase

5. Cervical lymphadenopathy $(\geq 1.5 \mathrm{~cm}$ diameter $)$, usually unilateral

As for $\mathrm{KD}$, similar to auto-immune disease in pathogenesis, it occurs as the result of dysregulated immune system [1]. Actually, no specific infectious agent was consistently associated with $\mathrm{KD}$, but viral etiology was considered for its pathogenesis [2]. This suggests that KD could be trigged by an acute viral infection, measles virus in our case, which was responsible for the abnormal immune response.

The diagnosis of classic or typical KD is based on the presence of $\geq 5$ days of fever associated to at least 4 of the 5 principal clinical features (Table 1) [6]. However, clinical features may not all be present at the same time, that's why a careful review of prior symptoms and signs is recommended [6]. In such cases, not only laboratory, but also echocardiographic findings, detecting coronary artery abnormalities, may be helpful. In fact, previous studies showed that abnormalities of liver function tests, non-severe cytolysis in our case, are frequently found in patients with acute KD and those are at higher risk for IVIG resistance [7].

As for KD treatment, which should be started without delay, it is based on IVIG at a high dose of $2 \mathrm{gr} / \mathrm{kg}$. The main goal of treatment is to reduce the development of coronary artery abnormalities [2]. Coronary artery abnormalities were reported in $20-25 \%$ of untreated children [8]. Besides IVIG, aspirin is used for its antiinflammatory activity at a dose of $80-100 \mathrm{mg} / \mathrm{kg} /$ day in the acute phase. It is followed by a dose of 3$5 \mathrm{mg} / \mathrm{kg} /$ day during the sub-acute phase for at least 6 8 weeks for its antithrombotic activity [1]. It is believed that a high dose of IVIG associated to aspirin could decrease the incidence of aneurysms to $3-5 \%$ and also shorten the duration of fever [8]. In our case, the association with a viral infection which is measles prevented the use of high dose of aspirin in order to avoid serious complications such as Reye syndrome [9].

Till now, there is no specific antiviral therapy approved for treatment of measles, which remains only supportive including antipyretics and fluids. The World Health Organization recommends the administration of vitamin A to all children with acute measles; since it was associated with decreased morbidity and mortality. It is administered once daily for two days at a dose of 50000,100000 and $200000 \mathrm{IU}$ for infants $<6$ months of age, aged from 6 to 11 months and $\geq 12$ months, respectively [10].

The disease evolution depends basically on the coronary artery abnormalities. In our case, the control echocardiography showed a reduction of the lumen diameter of the right coronary, which was reassuring. However, a long-term follow-up remains required.

\section{CONCLUSION}

Our case reported a febrile skin rash which hided another: KD was trigged by measles. Identifying the pathogenesis and the etiology of $K D$, might help to prevent it and to provide a documented treatment. Not to mention, both prompt diagnosis and treatment may avoid serious complications. That's why clinicians must be aware of the differential diagnosis between KD and measles, while keeping in mind that these two diseases can be associated, as well.

Author contributions: All authors contributed equally. All authors confirmed the final article.

Conflict of Interest: None to Declare

Source of funding: No funding

\section{REFERENCES}

1. Agarwal S, Agrawal DK. Kawasaki disease: etiopathogenesis and novel treatment strategies. Expert review of clinical immunology. 2017;13(3): 247-58. doi: 10.1080/1744666X.2017.1232165 PMid:27590181 PMCid:PMC5542821.

2. Dietz SM, van Stijn D, Burgner D et al. Dissecting Kawasaki disease: a state-of-the-art review. European journal of pediatrics. 2017; 176(8): 9951009. doi: 10.1007/s00431-017-2937-5 PMid: 28656474 PMCid:PMC5511310. 
3. Kim HS, Kim WD, Lee YH. Production and expression of Gro-alpha and RANTES by peripheral blood mononuclear cells isolated from patients with Kawasaki disease and measles. Journal of Korean medical science. 2003; 18(3): 381-6. doi: 10.3346/jkms.2003.18.3.381

PMid:12808325 PMCid:PMC3055045.

4. WHO. Immunization, Vaccines and Biologicals: New measles surveillance data for 2019. Available at:

https://www.who.int/immunization/newsroom/ measles-data-2019/en/ (Accessed: 19 May 2019).

5. Buonsenso D, Macchiarulo $G$, Supino $M C$, et al. Laboratory Biomarkers to Facilitate Differential Diagnosis between Measles and Kawasaki Disease in a Pediatric Emergency Room: A Retrospective Study. Mediterranean journal of hematology and infectious diseases. 2018; 10(1): e2018033. doi: 10.4084/mjhid.2018.033 PMid:29755710 PMCid: PMC5937975.

6. McCrindle BW, Rowley AH, Newburger JW, et al. Diagnosis, Treatment, and Long-Term Management of Kawasaki Disease: A Scientific Statement for Health Professionals from the American Heart Association. Circulation. 2017;135(17): e927-e99. doi: $10.1161 /$ CIR.0000000000000484
7. Keeling IM, Beran E, Dapunt OE. Kawasaki disease and hepatobiliary involvement: report of two cases. Italian journal of pediatrics. 2016; 42: 27. doi: 10.1186/s13052-016-0238-7 PMid:26951087 PMCid:PMC4782281.

8. Kuo HC, Lo MH, Hsieh KS, Guo MM, Huang YH. HighDose Aspirin is Associated with Anemia and Does Not Confer Benefit to Disease Outcomes in Kawasaki Disease. PloS one. 2015; 10(12): e0144603. doi: 10.1371/journal.pone. 0144603 PMid:26658843 PMCid:PMC4686074.

9. Noor A, Gradidge E. A Case of Reye Syndrome Caused by Influenza A Virus. The Ochsner journal. 2018; 18(4): 425-7. doi: 10.31486/toj.18.0098 PMid:30559633 PMCid:PMC6292460.

10. Bichon A, Aubry C, Benarous L, et al. Case report: Ribavirin and vitamin $A$ in a severe case of measles. Medicine. 2017; 96(50): e9154. doi: 10.1097/MD.0000000000009154 PMid:29390321 PMCid:PMC5815733. 